\title{
Visibilities and Invisibilities in Data Reuse: Video Records of Practice in Education
}

\author{
Elizabeth Yakel, Rebecca D. Frank, Kara Suzuka, Jasmine Smith
}

Note: This paper was published in Qualitative Research. Please cite the published version.

Citation: Yakel, E., Frank, R. D., Suzuka, K., \& Smith, J. (2021). Visibilities and Invisibilities in Data Reuse: Video Records of Practice in Education. Qualitative Research, 146879412110059. https://doi.org/10.1177/14687941211005955

\begin{abstract}
This article investigates data reuse or the secondary analysis of qualitative data, specifically video records of practice in education which are used to study the complex cognitive, social, and logistical issues involved in teaching and learning processes. It examines reuse through the lens of the invisibilities experienced by educational researchers who perform secondary analysis on video records of practice. Drawing on twenty-two in-depth interviews with educational researchers, we examine how they conceptualize secondary analysis of qualitative video data and cope with invisibilities in the data. For example, knowing the original research question was not sufficient: reusers needed to understand more about the intentionality of the data producer. They also sought more information on the reflexivity of the original researcher and how this influenced data production. Additionally, reusers discussed the creation of evidence from the video during secondary analysis as teaching and learning themselves entail invisible processes.

This work is licensed under a Creative Commons Attribution 4.0 International License (CC BY 4.0). http://creativecommons.org/licenses/by/4.0/

This research is funded by the Institute of Museum and Library Services \# LG-06-14-0122-14.

Elizabeth Yakel

School of Information, University of Michigan, USA

Rebecca D. Frank

School of Library and Information Science, Humboldt Universität zu Berlin, Germany

Kara Suzuka

College of Education, Curriculum Research \& Development Group, University of Hawai'i at Mānoa, USA

Jasmine Smith

Institute for the Study of the Ancient World (ISAW), New York University, USA
\end{abstract}




\section{Visibilities and Invisibilities in Data Reuse: Video Records of Practice in Education}

\section{Abstract}

This article investigates data reuse or the secondary analysis of qualitative data, specifically video records of practice in education which are used to study the complex cognitive, social, and logistical issues involved in teaching and learning processes. It examines reuse through the lens of the invisibilities experienced by educational researchers who perform secondary analysis on video records of practice. Drawing on twenty-two in-depth interviews with educational researchers, we examine how they conceptualize secondary analysis of qualitative video data and cope with invisibilities in the data. For example, knowing the original research question was not sufficient: reusers needed to understand more about the intentionality of the data producer. They also sought more information on the reflexivity of the original researcher and how this influenced data production. Additionally, reusers discussed the creation of evidence from the video during secondary analysis as teaching and learning themselves entail invisible processes.

\section{Introduction}

Data reuse or secondary analysis is plagued by gaps in data documentation and unknowns regarding the original data collection activities. In order to meaningfully reuse data, data reusers must overcome these and other challenges. This problem is even more acute in qualitative research, where the investigator is so closely linked to data production: the data producer is literally part of the instrumentation. Often, qualitative research involves a nuanced, reflexive, coconstruction of the data - or comprises a situated activity that only has meaning in a specific context (Bishop, 2007; Kwek and Kogut, 2015; Mauthner et al.,1998; Moore, 2007). In this article, we examine how data reusers perceive the invisibilities inherent in qualitative data, originally collected with other research aims, through an examination of educational researchers who reuse video records of practice to study the complex cognitive, social, and logistical issues involved in teaching and learning processes (Lewis 2007).

In education, records of practice are multimodal, providing aural and visual data; they are "detailed documentation of teaching and learning...taken directly from teaching and learning, without analysis, which enable (people) to look at practice" (Bass et al., 2002: 79). Records of practice encompass such documentation as lesson plans, seating charts, and student work, as well as photographs or video recordings of classroom activities. Video records of practice capture teachers leading lessons and/or students engaging in class discussions or small group 
and independent work. This article focuses on researchers who have sought and reused digital video records of practice to study educational activities, practices, and processes.

We focus on video records of practice for several reasons. First, video data provide both an audio and visual record of actions yet still reflect perspective and biases. As such, they require interpretation by the data producer, as well as by any subsequent data reusers, as to what those actions mean (Derry et al., 2010). Second, educational researchers have used video records of practice for over fifty years (e.g. Burleigh and Peterson, 1967). Until recently, individual person-to-person data sharing was the norm. However, over the last two decades there has been increased access to video data through online repositories (Baecker et al., 2007; Suzuka et al., 2018) thus increasing accessibility and usage levels. Third, as discussed by Hoeppe (2019) in relation to digital photographic images in astronomy, the infrastructure and medium of video records of practice is a critical dimension of the structuring and practice in this area of educational research. Finally, although video data make actions visible and audible (Lewis, 2007; Jewett 2012), as with any data they engender invisibilities which affect secondary analysis.

In this article, we consider how video records of practice and the infrastructures around research create or mediate invisibilities and visibilities during data reuse. We ask two research questions: (1) What types of invisibilities challenge reusers of video records of practice? and (2) When using video records of practice, how do data reusers construct knowledge and use video records of practice as evidence?

\section{Research as Invisible Work}

Knorr-Cetina (1999) argues that disciplines form epistemic cultures with specific norms and understandings for knowledge production, particularly around the construction and constitution of evidence. Knowledge construction is particularly interesting when scholars did not collect the data being used. The phenomenon of using data one did not collect is variously referred to as data reuse or secondary use. Researchers studying these phenomena employ the terms interchangeably and embrace different definitions. Common aspects of data reuse definitions include data previously created in research or practice (Heaton, 2008; Tuma, 2019); whether the secondary analysis must have a purpose different from the original study (Bishop and Kuula-Luumi, 2017; Faniel and Jacobson, 2010; Fielding and Fielding, 2000); and whether data reuse can involve the researchers involved in the original study (Faniel and Jacobsen, 2010). While the original researchers and the secondary data reusers face some of the same challenges, reusers contend with particular challenges. As Irwin and Winterton (2011) argue (italics original), "Primary analysts have a privileged relationship to the data they have generated, but do not necessarily have a privileged claim on the arguments which can be made from that data" (p. 8). In this article, we targeted data reusers who did not produce the original data in order to best examine how data are communicated over time and space.

For qualitative data reusers, knowledge production and the construction of evidence are particularly difficult since the motivations for the research as well as the specifics of data 
collection and analysis are often unclear. This problem of invisibility is compounded in qualitative research. Communicating the nuances of a study from the original researchers to later data reusers is more complex. There may also be epistemological concerns not overtly captured in the research questions or the design. Yet, an epistemological stance (e.g., positivist or constructivist) has implications for data reuse (Jones et al., 2018). Thus, some, such as Mauthner and her coauthors (1998), consider the problems of secondary analysis so severe they caution about reusing more than a study's research design. They are joined by Broom et al. (2009) who point to the situatedness of qualitative research and the high degree to which it is both context- and relationship-dependent, making reuse challenging. Finally, Moore (2007) cautions against attempting to recreate the context of the original research and proposes that reusers should plan to decontextualize qualitative data during reuse. Others note additional issues, such as the relationship between the original researcher and participants; the researcher's integral role as part of the instrumentation (Cassel, 2005; Fielding and Fielding 2000); and the co-construction of data by researchers and participants (Mauthner et al., 1998). Kwek and Kogut (2015) cite the "special relationship... between researcher and the research participant, or between researcher and research data, given the personal nature of data production for many researchers" (p. 17). Berger (2015) summarizes, "the worldview and background of the researcher affects the way in which he or she constructs the world, uses language, poses questions, and chooses the lens for filtering the information gathered from participants and making meaning of it, and thus may shape the findings and conclusions of the study" (p. 210).

Determinations of the usefulness of data for secondary analysis can be difficult since research practices are often invisible. In spite of all of these challenges, qualitative data reuse is possible while problematic as shown in accounts of reusing qualitative data. For example, Bishop (2007) concludes, "The experience of being able to probe in later interviews for themes that emerge early was constrained by not having data specific to my purpose and not being present at interviews" (Section 11.2). Bishop and Kuula-Luumi (2017) report a more positive experience of one qualitative data reuser who re-situated the data to answer new research questions.

Likewise, O'Connor and Goodwin (2010) performed secondary analysis on a set of interviews and then reinterviewed some of the original respondents 40 years later. Their study was greatly aided by the fact that they were able to find substantial information about the context of the original investigation through archival and published sources. Data producers' decisions about data creation and collection embody tacit knowledge that is often not captured and therefore cannot be shared later. Using archival sources, Métraux (1991) examined the development, codification, and teaching of research practice in bacteriology in the nineteenth century by analyzing of Alexandre Yersin's Berlin laboratory records. Yersin's reports show the conscious articulation of practice, both because the field was developing and because the researchers were anxious to disseminate their techniques more widely among scientists. Yersin's records were very detailed and the visibility of his process allowed for replication. This is not the norm for data reuse, both in terms of the depth of documentation and the desire to use the data for replication (as opposed to answering new research questions). Research documentation can be a poor indication for the actual methodological process (Latour and Woolgar, 1986) and a poor representation of scientific work (Lynch, 1985). Both Latour and Woolgar (1986) and Lynch 
(1985) showed that research design and methodological processes were difficult to capture, and the original research teams often negotiate how to best express their processes.

As Birnholtz and Beitz (2003) note, "knowledge transfer...is not simply a matter of sharing a set of instructions, but is a highly social process of learning practices that are not easily documented" (Birnholtz and Bietz, 2003: p. 340).

The invisibility of research design and the ensuing methods are borne out in the research on data reuse. Many studies have concluded that documentation of both the design and the actual methodology employed was critical for reuse, but often lacking (Faniel and Jacobsen, 2010; Wallis et al., 2013). Furthermore, different methodological details are important to different research communities. Birnholtz and Beitz (2003) found that "understanding the data for an [earthquake engineering] experiment, requires a detailed description of the experimental apparatus at the moment of testing, understanding HIVIAIDS study data requires knowledge of the study population and how it changed over time" (p. 346). While overall research practices around formulating a research design and executing a methodology are difficult to recreate, these are not the only aspects of research that are often invisible. Once data are collected, data cleaning, recoding, and analysis activities can also raise questions in the minds of data reusers (Faniel et al., 2012).

Disciplines deal with the invisibilities inherent in their data differently. Patel and Ball (2008) describe the adoption of new technologies in crystallography which captured and reproduced laboratory experiments, replacing laboratory notebooks that simply described an experiment and the results. Yet, even in crystallography scientists' concerns about the calibration of the machines and algorithms in the data processing software still generated questions about the resulting data. Edwards et al. (2011) have also shown how even the best intentioned efforts to summarize or describe data can cause confusion and fail to communicate the key aspects of the underlying data. They call this inability to smoothly and transparently transfer the knowledge about that data "science friction." In the sciences, data reusers are challenged by the tacit knowledge of the data producer (Collins, 2010; Zimmerman, 2008).

Visual data present more challenges and invisibilities. Lynch (1985) argues the creation of visual or graphic representations in science make the data knowable and analyzable. Other researchers have built on Lynch's work by analyzing the use of photographic images in science and data reuse (Hoeppe, 2019; Vertesi, 2015). Rijcke and Beaulieu (2014) note that the authority in functional magnetic resonance imaging (fMRI) brain scans is inseparable from their technical infrastructure. This is true of video records where concerns about the technical set up and framing "facilitate or undermine subsequent analysis" (Luff and Heath, 2012, p. 256) for both the original researcher and future data reusers (Blikstad-Balas, 2017). In video records of practice, for example, what lies just beyond the camera's view, what was left on the proverbial editing room floor, or simply not included for analysis or discussion has been shown to influence analysis (Derry et al., 2010).

The context of production of video data is particularly important. Tuma (2019) refers to vernacular video analysis as a type of data reuse performed on video resulting from everyday 
actions in naturalistic contexts. In Tuma's work, though, researchers are not involved in the production of police video. While video records of practice in education also fall into the category of vernacular video there is an important distinction between these and police data. Video records of practice are generally non-naturalistic data. As argued by Heaton (2008) nonnaturalistic data are intentionally "produced" for research purposes. Naturalistic data are personal or administrative actions or processes (e.g., diaries, family photographs, police video). While Tuma notes that in vernacular video analysis interpretation is the central activity and not production or processing, with non-naturalistic data, such as video records of practice, secondary analysis must take the production and post-processing into account.

While some, such as Korkiakangas (2010), have argued against the reuse of video data, it remains an essential tool for capturing, studying, and communicating about educational phenomena. Video records of practice depict the invisible activities of teaching and learning in situ in the classroom, which enhances their value as research data (Lewis, 2007): They can be watched multiple times, which can facilitate interpretation (Hadfield and Haw, 2012) and support close examination by single or multiple researchers using common or varied frameworks (Sammons and Lindorff, 2018) which are both quantitative and qualitative in design (Bell et al., 2019). Research groups commonly view and interpret the videos together to examine teaching and learning methodologies and behaviors (Andersson and Sørvik, 2013; Burleigh and Peterson, 1967). This includes generative work to develop and refine analytic constructs (Schoenfeld, 2013); efforts toward improving the reliability of observations, interpretations, and coding (Derry, 2007; Derry et al., 2010; Learning Mathematics for Teaching Project, 2011); as well as endeavors to validate frameworks (Sammons and Lindorff, 2018). Video records of practice are also being used to enable distributed, collaborative research (Goldman, Pea, Barron and Derry, 2014) —as well as new, diverse research efforts through data sharing and reuse. An early example of this type of distributed collaborative effort is the TIMSS 1995 and 1999 Video studies (Stigler, Gonzales, Kawanaka, Knoll and Serrano, 1999; Hiebert et al., 2005). Still, identifying mechanisms to facilitate the reuse of video records of practice is important to the field as they are costly and difficult to produce (Frank et al., 2017; Bell et al., 2019).

\section{Research Methods}

This research is based on forty-four semi-structured in-depth interviews with video data reusers in the field of education, principally focusing on mathematics education research and teacher education. To recruit interviewees, we consulted research literature to identify scholars who reused video records of practice, attended academic conferences, and asked participants to recommend others we should interview. Using convenience and snowball sampling techniques, we identified academic researchers who utilized video records of practice as well as pre-service and in-service teacher-educators engaged in teacher preparation programs or conducting professional development activities reusing records of practice.

In this article, we focus on twenty-two interviewees who reused video records of practice in the context of research. Of those twenty-two interviewees, thirteen were faculty members, one a 
postdoctoral researcher, five doctoral students, and three professional research specialists. Nineteen had collected their own data, in addition to reusing video data collected by others. Interviewees described their research goals as: developing and testing theories of teaching and learning, classification systems for educational practices, and tools for video analysis; analyzing cultural and/or socioeconomic factors that influence student learning, student thinking, reasoning, and/or problem-solving; and understanding teacher-student interactions, questioning, and/or argumentation. They used a variety of specific qualitative research methods such as narrative analysis, discourse analysis, grounded theory, phenomenology, and critical theory. The participants' research motivations varied, some were aligned with those of original studies and others were not. All interviews were recorded and transcribed. Transcripts were then analyzed with NVivo using a set of codes that were generated based on themes from the literature, our interview protocol, and themes that arose during the interviews themselves. Codes addressed topics relating to data sharing and reuse, including contextual information needed for data reuse; teaching and research; and visibilities and invisibilities. Transcripts were coded by two team members working in tandem on the same transcript until they reached an acceptable level of interrater agreement using Scott's pi, a statistic measuring interrater reliability for coding textual data (Holsti, 1969). For the twenty-two research-focused interviews we achieved a Scott's pi score of 0.712 . This study was reviewed by the [institution] Institutional Review Board and deemed exempt [study number].

\section{Findings}

Our results examine how educational researchers negotiate the reuse of video records of practice. We segment our findings into issues related first to data production examining: 1) the intentionality and role of the data producer in research design decisions during data production, 2 ) the data producer as instrument, and 3) the construction of evidence. Then we turn to the construction of evidence. Through these three dimensions, we highlight how visibilities aid and invisibilities challenge data reuse.

\subsection{Data Production}

In this section, we highlight two invisible aspects of data production: data producers' intentions concerning study conceptualization and their rationales for research design decisions. Reusers deemed this information critical when determining whether and how the data could be reused.

\subsubsection{Intentionality}

The intentionality of the data producer was a major issue in reuse. Intentionality was the word specifically used by our interviewees to sum up this need for broader context. In the first example (Researchers 022 and 035), the researchers found the way in which the video was filmed - the focus and framing-provided affordances for a variety of later research inquiries into learning and teaching. In the second (Researchers 039 and 002), the researchers worried about not fully knowing the intentionality of the data producer and the potential for subsequent overreach or misuse of data to understand learning processes in the classroom. In the third, 
Researcher 041 , asked about both the identity of the data producer and his or her intentions (context) as important markers for trusting the data.

In each of three examples, data reusers considered how data producers' intentions impacted their research questions. Researcher_022 noted: "Some of these older clips... are pretty nice in terms of zooming in on a student's work, or then panning up to the teacher, and it's sort of very clearly, intentionally filmed." The dual focus on the students and the teacher allowed her to study both teaching and student learning. Likewise, Researcher_035 concluded, “...when you're repurposing a collection, you're constrained by the videography of the time as well as the focus for whatever the person was that was collecting it, what their focus was at that time."

Other participants also reflected on the importance of the original research questions.

Researcher_039 felt confident in her use of one collection of video records of practice because, "It's just that I know the rationale behind the creation of these videos so I know they match with what I'm trying to achieve, so it's easier that way." In contrast, Researcher_002 questioned reuse due to the original conceptualization behind the dataset, "And I think that if you're using someone else's data, that you need to be very cognizant and transparent about the limitations of that data because those data were not collected with the questions that you're asking." She continued by raising ethical concerns about whether a new use would be appropriate, or if reuse would involve making the data fit analyses for which they were not originally intended, and emphasized the importance of understanding the perspective and focus of the data collection:

'When you read that description...It's not really clear what the original questions or reasons for filming the videos are, and I think that that's also really important. So, I would wanna know opening a library, what are the original questions that frame the collection of this data, so I can understand the perspective of the filming, the perspective of the audio recording. So, I can maybe kind of... understand what wasn't captured as well as what was captured, that would really be important to me.' (Researcher_002)

The inextricable link between research design and which phenomena are captured, and the original research questions on the other, was aptly summed up by Researcher_041, "...It's important to keep in mind the intentions and the perspectives and the agendas of the researchers who originally curated data so that when you're interpreting it for other purposes you can take that perspective into account." In each of these examples, the data producers' intentions - and the degree to which data reusers could understand those intentions influenced data reuse.

\subsubsection{Data Producer as Instrument}

Qualitative research is heavily dependent on the data producer's positionality, as an extension of the instrumentation (Cassell, 2005; Rubin and Rubin, 2005). In the case of video records of practice the concept of data producer as instrument, or lens, through which data is interpreted holds a double meaning.. Figuratively the data producer's epistemology and positionality affects the research design, and the data producer literally directs the camera's lens and microphone toward certain activities. As Researcher_002 states, "I want to know what your lens allowed you to see and what your lens didn't allow you to see as a researcher." In one instance, Researcher_002 asked the original data producer to collaborate to access fuller design documentation and what he characterized as "all that insider information." Researcher_035, who was a data producer as well as a data reuser reflected on the production process,

'...[W]hen you're in a classroom... even though you try to be as independent and neutral 
as possible, you still have a certain lens that you're still bringing to it and that shows up in your field notes. We always try to use video regardless because I think it's more a good way to get reliability that went on in the session and really captures the complexity that's really going.'

Consequently, reusers of video records of practice wanted to know the identity of the data producer. Researcher_018 cited trust, "...If I was using it for research I would care about, who videoed it: Why? What was the context?...Yeah, just the trustworthiness I guess." Two other interviewees (Researchers 001 and 003) identified one dataset, produced by a researcher who was also the teacher featured in the dataset, as being highly documented with very transparent research designs. They noted how the lesson planning documentation, teacher's journals, and other records of practice accompanying the videos shed light on the teacher's classroom activities as well as the researcher's positionality. Researcher_001 concluded that the visible nature of the dataset enabled data reusers to ask more types of research questions.

Other participants discussed how positionality affected the data producer's research design, analysis, and interpretation. Researcher_002 was concerned about the positionality and influence of research teams,

'I really struggle with the invisibility of researchers, and I wanna know whose data I'm reading...I think it's an issue of the principals' but also the project teams', like I wanna know that their... I think it doesn't matter if you're studying critical issues or if you're studying feedback and practice, that like so many of our instruments I'm coming to know digging into it have been normatized to either male populations or white populations or middle class populations, but that's not transparent and so I think there's something about knowing who's doing the work that will at least allow us to ask those questions even if everything is totally on surface, like completely fine and they really did the best that they could in the field and gathered all the data in very professional ways, using the best tools and best practices, that's fine, but at least I know to ask those questions.'

Research objectives and design decisions-whether they are conceptual (research questions) or technical (video camera and microphone placement) - are both important to data reusers. However, this "insider information" is often unarticulated and invisible in video records of

practice. For the data reusers we interviewed, this left significant questions about the appropriateness of data reuse in their minds. Although one participant ended up collaborating with the original data producer in her new study, others reflected on major questions about the data as they reused them.

\subsection{The Construction of Evidence}

In addition to the invisibilities inherent in the data, the actual evidence sought after by data reusers is also invisible. Teaching and learning are subtle and, in many cases, invisible processes. Thus, researchers reusing video records of practice are often looking for nuanced spoken cues or body language. Our interviewees described the difficulty of both capturing and then recognizing the invisible work of teaching and learning.

Researcher_004 argued that making teaching and the work of teachers depicted in video records of practice visible was key to the usefulness these data: "The more visible the teaching 
is and the work that the teacher's involved in is, the more useful it is." Student learning and teachers' practices, however, are not always visible. Researcher_003 described how overt behaviors in the video signaled evidence of implicit student learning for her team: "These are the three student learning practices: Listening, speaking publicly, and making and using records...So you can't see listening, so we code for 'Seeking evidence' and for 'Citing evidence."”

The nuanced nature of teaching can lead to varied interpretations and analytic judgements by researchers that arise from their perspectives and biases. Researcher_022 discussed how her research team works to standardize their analyses and minimize bias during analysis:

'... YY]ou also participate in regular calibration sessions where our team here has sort of watched and master scored a short clip of instructions, and then we assign all of our raters to score those clips. And then we have a weekly webinar where we sort of go over codes in which, or clips in which, the raters are straying from master scores on the instruments.'

Like teaching, learning is a much nuanced activity. Researcher_040 talked about the difficulty in discerning and finding evidence of learning, but noted how video records of practice have the potential to make learning visible:

'[I]t's really hard to determine what was the student's idea and what was the student's idea from the reading. Were they summarizing the reading or were they kind of incorporating themselves in this? I felt with the video it really kind of opened up what is the student thinking about this concept: What are they believing? What are they seeing and so forth. I think it's really been useful in terms of both teaching, research, and understanding what people are thinking or so forth instead of are they really just parroting back what someone told me?'

While interpretation is a factor in all research, in qualitative research the interpretivist process is a central feature of data analysis. Researchers analyze more than what is said or depicted on video, but rather are interested in latent, covert meaning. One researcher who frequently engaged in collaborative data analysis described the process of jointly analyzing video records of practice as generative work:

'We'll watch for a little while, somebody typically stops (the video), we talk, and we might go back and re-watch it, then, more carefully. There's a lot of that kind of work that's very generative, and then there's also times when we get to a point where we decide to code on the video.' (Researcher_004)

In this example, the researchers were actively constructing meaning, as well as evidence, while they viewed the video. The combination of individual judgments and checking interpretations with colleagues helped verify both the evidence and their interpretations. 
Analyzing teaching also presents analytical and evidential problems. The goal of the teacher on the video becomes paramount and understanding this intent may require additional information, such as a lesson plan or post-class interview. The invisible actions around teaching and the evidence required to understand a teacher's goals were Researcher_001's focus: "Like what the teacher is thinking about and how did they plan the lesson, so there are lots of complementary artifacts you want to be able to have available to interpret what you're seeing as well." Furthermore, researcher biases and expectations can obscure-or make invisible-certain facets of what there is to see in the video. This becomes particularly salient when, for example, the culture of those in the classroom is unfamiliar to or inadequately understood by the observer. Under these circumstances, key facets of teaching or evidence of student learning might be invisible to the observer. Researcher_002 reflected on such biases in how people view the teaching and learning found at "many low resource schools," where good learning takes place but is often not seen or recognized as such:

'I didn't see very many low resource schools in the videos that I looked at...some of that teaching wouldn't have been certified because of different cultural norms. I taught in a low resource school and my classroom would have looked incredibly loud and chaotic to an outsider. There was a lot of good learning going on, and I have a lot of evidence to back that up, but someone would have come into that room who didn't have that context and most likely made some assumptions about what was happening and who was in that room and why it was happening that weren't at all true. Those kids wrote newspaper articles that got published in local news. They were doing really good work.'

The implication is that certain types of excellent teaching and student learning are invisible due to our assumptions about what this means. As a result, they can easily be missed in video recordings or not even recorded in the first place.

Such examples of teaching and learning continue to be invisible and unseen. Researcher_002 continued and articulated the "tension" she experienced when applying standardized 'tags' or codes to a set of videos with her team:

'I was constantly watching mathematics videos for elementary school mathematics ... And I would be watching something that...would've been considered a discussion (using the team's definition). So you could label it a discussion. The terms of talk were centered around something, but you can see by how the video clip was unfolding that there's no intentionality for discussion there...if I had been a teacher educator or field instructor in that room, I would not have called that a discussion. But the tag as it was written allowed me to call it a discussion. So you had a little bit of tension there.'

For this researcher, the way this facet of classroom practice was defined and identified during the data analysis process obscured key dimensions of teaching practice that she considered to be important, leading her to identify this and other classroom activities in ways she found problematic. 
Indeed, getting to the point where a researcher can even perform this type of analysis on video records of practice can be difficult. Researchers often viewed hours of video before deciding whether the data were a good fit for their research questions, as noted by Resarcher_003:

'Looking through video is very time-consuming. Then, comparing the video to the transcripts is very time-consuming. I think looking at video can be more time-consuming than looking at transcripts because sometimes you have to watch it over and over and over to hear, get the whole sense of what's happening.'

Evidence of teaching and learning can be made visible over time, or may remain invisible. This can be due to something as simple as the focus of the video camera. Researcher_004 explained that the quality of video production, including the focus of the camera, can have a significant impact on what kind of analysis data reusers are able to do:

'There may be just tracking on the one and not the other: either the students or on the teacher. But with not enough regard for the other, that we can make sense of the interactions that are occurring, or where the teaching is... You know, just not of the kind that makes things visible... Having video of just the lecture is not very supportive with the kind of analysis that we're wanting to do.'

Several interviewees discussed the difficulties of capturing data and making claims in light of the invisibilities inherent in only seeing parts of a larger video or seeing a video without the larger context of the classroom in which the events took place. For example, Researcher_013 articulated a common feeling among the interviewees that something was happening in the classroom just beyond the video invisible to them.

'But I think camera placement kind of toward the front of the room, so we could see everyone at the same time. Sometimes it was a little hard to see who was getting called on, who was speaking and also when things move into small groups, right? ... Like how do you, without having eight different cameras in the room for all the different groups, how do you capture data on what's happening writ large in each small groups for itself?'

The use of video to uncover invisible concepts was also mentioned as a way to bolster one's evidence beyond simply relying on a written description or transcript of a recorded event. Researcher_035 reflected:

'I think the way it used to be done before video was accessible this way is you would just describe the video, and maybe you'd provide a transcript, like, "Oh, look. This is what happened, and this is what I thought happened." Now, you can describe it and then you can provide a link where people can actually look at what actually happened. I think that's really powerful because to see it, for researchers, for teachers, for teacher educators, for people that are maybe using your article that you've written based on this research in their own research to be able to actually click on it and see the link, visit the analytic or visit the video and be able to look at it themselves. I think is really, really powerful.'

In this section we presented, among other things, a critical examination of how different researchers think about and interrogate video records of practice as evidence, analyze that 
evidence, and reach conclusions for publications. While video records of practice capture physical classroom activities, they only get researchers so far in understanding the cognitive process of teaching and learning. Because teaching and learning are both processes that are difficult to make visible, researchers want to do more than create a description of what happened. Rather, they want to find ways to render these often invisible processes visible through their research processes.

\section{Discussion}

Researchers reusing video records of practice were reflective about their approaches to secondary analysis. In particular, they were very interested in identifying the intentionality of the data producer and how that shaped the original research study. Data reusers employed information about intentionality to make decisions about whether video data were relevant for their specific research goals but more importantly saw it as a way to determine how the data could appropriately and ethically be used as evidence. Our participants also described how they constructed evidence from video of 'invisible' educational practices and processes, along with the difficulties they faced in determining whether or not they could find evidence addressing their research questions in the records of practice. In addition, data reusers discussed how the evidential value of the video records of practice would be augmented by additional information to improve the visibility of the research practices that produced the data-such as information about the intent of the original data producer or the specifics of the research design. In our discussion, we focus on the issues raised by our interview respondents concerning the study of invisible processes (e.g., teaching and learning) and the aspects of the original research practices that reusers most needed to understand.

Teaching and learning are hard to analyze. Video records of practice capture classroom activities so that researchers can attempt to discern nuances in teaching practices and student learning. However, this does not eliminate the duality between capture and analysis; Jewitt (2012), quoting Pink, aptly states, "things become visible because of how we see them rather than simply because they are observable" (p. 11). Still, teaching involves agency and intentionality on the part of the teacher and student learning is a highly cognitive activity. None of these are explicitly captured on video, rather these implicit and nuanced phenomena emerge during analysis and the construction of evidence.. In reference to reuse of video records of practice, Derry et al. (2010) noted that the "ability to communicate and share across research groups requires some degree of commonality among practices and tools." However, standardization only enables reuse to a certain extent. Educational researchers using qualitative methods also needed to understand aspects of the video data in order to construct evidence, such as the research intentions of the data producers, were translated into the video as well as the ensuing documentation. This aligns with Yoon (2014a) who found that qualitative data reusers heavily rely on the original data producers for information vital for successful secondary analysis. However, Bishop and Kuula-Luumi (2017) disagree. They cite the Finnish Data Service which does not collect much information at the project level but strives to document the data, "This level of documentation seems to be sufficient for many users' requirements" (p. 9). 
This discrepancy may also have to do with the media of the data, as Bishop and Kuula-Luumi focused on interview rather than video data.

The nature of the invisibility of the original data producers' research practices may be disciplinary or media dependent. Hoeppe (2019) argues that while image media are fixed, interpretations are not. When constructing evidence, our interviewees were very interested in the "fixing" and they viewed the video production strategy as providing additional insight into the original researcher's intent as well as important information for determining whether a collection of records of practice was relevant for their study and value of any evidence it might yield. The ability to capture invisible phenomena does not necessarily capture the meaning behind them or eliminate the invisibilities in the context represented in the video (Jewitt 2012).

\section{Conclusion}

In 1998, Heaton called for more research into qualitative data reuse. Over twenty years later, there is still substantial controversy about qualitative data reuse and much we do not understand about overcoming the challenges and facilitating this type of data reuse. This article seeks to bridge this gap. Previous research has provided individual accounts of the challenges reusing qualitative data (Bishop and Kuula-Lummi, 2017; Broom, et al., 2009) or examined qualitative researchers from different disciplines reusing data more generally (Yoon, 2014b). Although there are studies of data reusers using qualitative data (Bishop, 2009; Corti and Fielding, 2016), few examine qualitative video data (Frank et al., 2018). Thus, this article is the first to present an in-depth look at multiple researchers in one discipline reusing a specific type of qualitative data. Future research will determine how the issues our participants experienced reusing video records of practice are distinct or emblematic of the challenges faced by researchers performing secondary analysis of qualitative data.

\section{Declaration of Conflicting Interests}

The Author(s) declare(s) that there is no conflict of interest.

\section{Funding}

The author(s) disclosed receipt of the following financial support for the research, authorship, and/or publication of this article: This work was supported in part by a grant from the Institute for Museum and Library Services \# LG-06-14-0122-14.

\section{References}

Baecker RM, Fono D and Wolf P (2007) Toward a Video Collaboratory. In: Video Research in the Learning Sciences. Lawrence Erlbaum Associates, pp. 461-478. 
Bass H, Usiskin Z, Burrill G, et al. (eds) (2002) Studying Classroom Teaching as a Medium for Professional Development Proceedings of a U.S.-Japan Workshop. Washington, DC:

National Academy Press. Available at: http://www.nap.edu/catalog/10289.html (accessed 19 March 2015).

Bell CA, Dobbelaer MJ, Klette K, et al. (2019) Qualities of Classroom Observation Systems. School Effectiveness and School Improvement 30(1). Routledge: 3-29. DOI: 10.1080/09243453.2018.1539014.

Berger R (2015) Now I See It, Now I Don't: Researcher's Position and Reflexivity in Qualitative Research. Qualitative Research 15(2). SAGE Publications: 219-234. DOI: 10.1177/1468794112468475.

Birnholtz JP and Bietz M (2003) Data at Work: Supporting Sharing in Science and Engineering. In: GROUP '03 Proceedings of the 2003 international ACM SIGGROUP conference on supporting group work, Sanibel Island, FL, 2003, pp. 339-348. ACM. DOI: 10.1145/958160.958215.

Bishop L (2007) A Reflexive Account of Reusing Qualitative Data: Beyond Primary/Secondary Dualism. Sociological Research Online 12(3): 121-141. DOI: 10.4135/9781446268544.

Bishop L (2009) Ethical Sharing and Reuse of Qualitative Data. Australian Journal of Social Issues 44(3): 255-272. DOI: 10.1002/j.1839-4655.2009.tb00145.x.

Bishop L and Kuula-Luumi A (2017) Revisiting Qualitative Data Reuse: A Decade On. SAGE Open 7(1): 215824401668513. DOI: 10.1177/2158244016685136.

Blikstad-Balas M (2017) Key Challenges of Using Video When Investigating Social Practices in Education: Contextualization, Magnification, and Representation. International Journal of Research \& Method in Education 40(5). Routledge: 511-523. DOI: 10.1080/1743727X.2016.1181162.

Broom A, Cheshire L and Emmison M (2009) Qualitative Researchers' Understandings of Their Practice and the Implications for Data Archiving and Sharing. Sociology 43(6): 11631180. DOI: $10.1177 / 0038038509345704$.

Burleigh JC and Peterson HW (1967) Videotapes in Teacher Education. The Elementary School Journal 68(1): 35-38. DOI: https://doi.org/10.1086/460406. 
Cassell C (2005) Creating the Interviewer: Identity Work in the Management Research Process. Qualitative Research 5(2): 167-179. DOI: 10.1177/1468794105050833.

Collins HM (2010) Tacit and Explicit Knowledge. Chicago, IL: University of Chicago Press. Available at: https://press.uchicago.edu/ucp/books/book/chicago/T/bo8461024.html (accessed 11 May 2020).

Corti L and Fielding N (2016) Opportunities From the Digital Revolution: Implications for Researching, Publishing, and Consuming Qualitative Research. SAGE Open 6(4): 215824401667891. DOI: $10.1177 / 2158244016678912$.

Derry SJ (2007) Guidelines for Video Research in Education: Recommendations from an expert panel. University of Chicago, Chicago, IL: Data Research and Development Center.

Derry SJ, Pea RD, Barron B, et al. (2010) Conducting Video Research in the Learning Sciences: Guidance on Selection, Analysis, Technology, and Ethics. Journal of the Learning Sciences 19(1): 3-53. DOI: 10.1080/10508400903452884.

Edwards PN, Mayernik MS, Batcheller AL, et al. (2011) Science Friction: Data, Metadata, and Collaboration. Social Studies of Science 41(5): 667-690. DOI: 10.1177/0306312711413314.

Faniel IM and Jacobsen TE (2010) Reusing Scientific Data: How Earthquake Engineering Researchers Assess the Reusability of Colleagues' Data. Computer Supported Cooperative Work 19(3-4): 355-375. DOI: 10.1007/s10606-010-9117-8.

Faniel IM, Kriesberg A and Yakel E (2012) Data Reuse and Sensemaking Among Novice Social Scientists. Proceedings of the American Society for Information Science and Technology 49(1): 1-10. DOI: 10.1002/meet.14504901068.

Fielding NG and Fielding JL (2000) Resistance and Adaptation to Criminal Identity: Using Secondary Analysis to Evaluate Classic Studies of Crime and Deviance. Sociology 34(4): 671-689. DOI: $\underline{10.1177 / S 0038038500000419}$.

Frank RD, Chen Z, Crawford E, et al. (2017) Trust in Qualitative Data Repositories. Proceedings of the Association for Information Science and Technology 54(1): 102-111. DOI: $10.1002 /$ pra2.2017.14505401012. 
Frank RD, Tyler ARB, Gault A, et al. (2018) Privacy Concerns in Qualitative Video Data Reuse. International Journal of Digital Curation 13: 47-72. DOI: 10.2218/ijdc.v13i1.492.

Goldman R, Pea R, Barron B, et al. (2014) Video Research in the Learning Sciences. Routledge.

Hadfield M and Haw K (2012) Video: Modalities and Methodologies. International Journal of Research \& Method in Education 35(3): 311-324. DOI: 10.1080/1743727X.2012.717434.

Heaton J (1998) Secondary Analysis of Qualitative Data. Social Research Update (22). Available at: https://sru.soc.surrey.ac.uk/SRU22.html (accessed 8 July 2020).

Heaton J (2008) Secondary Analysis of Qualitative Data: An Overview. Historical Social Research / Historische Sozialforschung 33(3 (125)). GESIS - Leibniz-Institute for the Social Sciences, Center for Historical Social Research: 33-45.

Hiebert J, Stigler J, Jacobs JK, et al. (2005) Mathematics Teaching in the United States Today (And Tomorrow): Results from the TIMSS 1999 Video Study. Educational Evaluation and Policy Analysis 27(2): 111-132.

Hoeppe G (2019) Medium, Calculation, Play: On Digital Images in Scientific Practice. Social Studies of Science 49(5): 758-784. DOI: 10.1177/0306312719871356.

Holsti OR (1969) Content Analysis for the Social Sciences and Humanities. Reading, M.A.: Addison-Wesley Pub. Co.

Irwin S and Winterton M (2011) Debates in Qualitative Secondary Analysis: Critical Reflections. Timescapes Working Paper Series 4, Working Paper. Available at: https://timescapesarchive.leeds.ac.uk/wp-content/uploads/sites/47/2020/07/WP4-March-2011.pdf.

Jewitt C (2012) An Introduction to Using Video for Research. Working Paper. London, U.K.: National Centre for Research Methods. Available at: http://eprints.ncrm.ac.uk/2259/4/NCRM workingpaper 0312.pdf.

Jones K, Alexander SM, Bennett N, et al. (2018) Qualitative Data Sharing and Re-Use for Socio-Environmental Systems Research: A Synthesis of Opportunities, Challenges, Resources and Approaches. Available at: https://doi.org/10.13016/M2WH2DG59 (accessed 8 November 2019). 
Kane TJ, McCaffrey D, Miller T, et al. (2013) Have We Identified Effective Teachers? Validating Measures of Effective Teaching Using Random Assignment. Bill \& Melinda Gates Foundation. Available at: http://k12education.gatesfoundation.org/resource/have-weidentified-effective-teachers-validating-measures-of-effective-teaching-using-randomassignment/ (accessed 15 December 2020).

Knorr-Cetina K (1999) Epistemic Cultures: How the Sciences Make Knowledge. Cambridge, Mass.: Harvard University Press. Available at: http://books.google.com/books?id=WFEeib0Q9L0C\&lpg=PP1\&pg=PP1\#v=onepage\&q\&f= false.

Korkiakangas TK (2014) Challenges in Archiving and Sharing Video Data: Considering Moral, Pragmatic, and Substantial Arguments. Journal of Research Practice 10(1). Available at: https://core.ac.uk/reader/268476352 (accessed 8 July 2020).

Kwek D and Kogut G (2015) Knowledge of Prior Work and Soundness of Project: A Review of the Research on Secondary Analysis of Research Data. Technical Report, Office of Education Research. Singapore: National Institute of Education.: 36.

Latour B and Woolgar S (1986) Laboratory Life: The Construction of Scientific Facts. Princeton University Press.

Learning Mathematics for Teaching Project (2011) Measuring the Mathematical Quality of Instruction. Journal of Mathematics Teacher Education 14(1): 25-47. DOI: 10.1007/s10857-010-9140-1.

Lewis JM (2007) Teaching as Invisible Work. Ph.D. University of Michigan, United States -Michigan. Available at:

http://search.proquest.com.proxy.lib.umich.edu/pqdtlocal1006034/docview/304847284/ab stract/23D0903E72A74389PQ/1 (accessed 19 October 2015).

Luff P and Heath C (2012) Some 'Technical Challenges' of Video Analysis: Social Actions, Objects, Material Realities and the Problems of Perspective. Qualitative Research 12(3). SAGE Publications: 255-279. DOI: 10.1177/1468794112436655.

Lynch M (1985) Art and Artifact in Laboratory Science: A Study of Shop Work and Shop Talk in A Research Laboratory. London: Routledge and Keegan Paul. Available at: http://doi.wiley.com/10.1111/1467-9566.ep11347044 (accessed 18 October 2015). 
Mauthner NS, Parry O and Backett-Milburn K (1998) The Data are Out there, or are They? Implications for Archiving and Revisiting Qualitative Data: Sociology. CAMBRIDGE UNIVERSITY PRESS. DOI: 10.1177/0038038598032004006.

Métraux A (1991) Reaching the Invisible: A Case Study of Experimental Work in Microbiology (1880-1900). In: Strauss AL and Maines DR (eds) Social Organisation and Social Process. Essays in Honor of Anselm Strauss. New York, N.Y.: Gruyter, Walter de, \& Co., pp. 249-260.

Moore N (2007) (Re)using Qualitative Data? Sociological Research Online 12(3). DOI: 10.5153/sro.1496.

O'Connor H and Goodwin J (2010) Utilizing Data from a Lost Sociological Project: Experiences, Insights, Promises. Qualitative Research 10(3). SAGE Publications: 283298. DOI: $10.1177 / 1468794110362875$.

OECD (2020) Global Teaching InSights: A Video Study of Teaching. Paris: OECD Publishing. DOI: $10.1787 / 20 \mathrm{~d} 6 \mathrm{f} 36 \mathrm{~b}-\mathrm{en}$.

Patel M and Ball A (2008) Challenges and Issues Relating to the Use of Representation Information for the Digital Curation of Crystallography and Engineering Data. International Journal of Digital Curation 3(1): 76-88. DOI: 10.2218/ijdc.v3i1.43.

Rijcke S de and Beaulieu A (2014) Networked Neuroscience: Brain Scans and Visual Knowing at the Intersection of Atlases and Databases. In: Coopman C, Vertesi J, Lynch M, et al. (eds) Representation in Scientific Practice Revisited. The MIT Press, pp. 131-152. DOI: 10.7551/mitpress/9780262525381.003.0007.

Rubin H and Rubin I (2005) Qualitative Interviewing: The Art of Hearing Data. 2nd ed. 2455 Teller Road, Thousand Oaks California 91320 United States: SAGE Publications, Inc. DOI: $\underline{10.4135 / 9781452226651 .}$

Sammons P and Lindorff A (2018) Observing Effective Mathematics Teaching: A Review of the Literature. Education Development Trust. Available at: https://www.educationdevelopmenttrust.com/CMSPages/GetFile.aspx?nodeguid=c230397 c-a9da-4ffa-be6a-15be1cbae0cc\&lang=en-GB/.

Schoenfeld AH (2013) Classroom Observations in Theory and Practice. ZDM 45(4): 607-621. DOI: $10.1007 / \mathrm{s} 11858-012-0483-1$. 
Stigler JW, Gonzales P, Kawanaka T, et al. (1999) Videotape Classroom Study: Methods and Findings from an Exploratory Research Project on Eighth-grade Mathematics Instruction in Germany, Japan, and the United States. 99-074, Research and Development Report. The TIMSS US Department of Education, Office of Educational Research and Improvement, National Center for Education Statistics. Available at: https://nces.ed.gov/pubs99/1999074.pdf.

Suzuka K, Frank R, Crawford E, et al. (2018) Video Re-Use in Mathematics Teacher Education. In: Society for Information Technology \& Teacher Education International Conference, 26 March 2018, pp. 329-336. Association for the Advancement of Computing in Education (AACE). Available at: https://www.learntechlib.org/primary/p/182544/ (accessed 7 May 2020).

Tuma R (2019) Vernacular Video Analysis - Communicative Construction of Evidence in Police Work. Society Register 3(1): 137-162. DOI: 10.14746/sr.2019.3.1.08.

Vertesi J (2015) Seeing Like a Rover: How Robots, Teams, and Images Craft Knowledge of Mars. Chicago ; London: The University of Chicago Press.

Wallis JC, Rolando E and Borgman CL (2013) If We Share Data, Will Anyone Use Them? Data Sharing and Reuse in the Long Tail of Science and Technology. PLoS ONE Nunes Amaral LA (ed.) 8(7): e67332. DOI: 10.1371/journal.pone.0067332.

Yoon A (2014a) End Users' Trust in Data Repositories: Definition and Influences on Trust Development. Archival Science 14(1): 17-34. DOI: 10.1007/s10502-013-9207-8.

Yoon A (2014b) "Making a Square Fit into a Circle": Researchers' Experiences Reusing Qualitative Data. Proceedings of the American Society for Information Science and Technology 51(1): 1-4. DOI: 10.1002/meet.2014.14505101140.

Zimmerman AS (2008) New Knowledge from Old Data: The Role of Standards in the Sharing and Reuse of Ecological Data. Science, Technology \& Human Values 33(5): 631-652. DOI: $10.1177 / 0162243907306704$. 